\title{
Quantifying the Effect of Substrate Compaction on Root Development in Cover Systems
}

\author{
A.B. Fourie School of Civil and Resource Engineering, The University of Western Australia, Australia \\ M. Tibbett Centre for Land Rehabilitation, The University of Western Australia, Australia \\ T. Worthington Centre for Land Rehabilitation, The University of Western Australia, Australia
}

A.E. King The University of Western Australia, Australia

\begin{abstract}
Vegetation is understandably an integral component of the cover systems used on most tailings and waste rock disposal facilities. The soil in these covers is usually placed and spread using earthmoving equipment such as trucks, scrapers, dozers and front-end loaders. Trafficking of this equipment can easily result in a high degree of compaction of the cover soil, which may compromise the ability of roots to develop laterals or penetrate to adequate depth. Poor root development may curtail the vegetation survival and growth, and thus reduce the effectiveness of the cover system.

Soil was collected from the stockpile of a candidate cover soil at a tailings storage site in Western Australia. The soil was compacted according to the relevant Australian Standard in $50 \mathrm{~cm}$ long, $10 \mathrm{~cm}$ diameter PVC growth columns. Three compaction configurations were used, namely high, low and stratified, in a replicated root penetration experiment. Pre-germinated seeds of Acacia celastrifolia were planted into each column and grown in a temperature regulated glasshouse facility. At 40 days and 60 days after detection of first growth, soil was extruded from the cylinders and the root architecture defined using a WinRHIZO scanner and software.

The paper illustrates that the (limited) available literature on the effect of compaction density on root development is simplistic and can be unnecessarily restrictive if adhered to blindly. The importance of working within a framework that contextualizes the degree of compaction, such as is used in engineering earthworks, is shown to be a key factor in interpreting the results of this type of study.
\end{abstract}

\section{Introduction}

Many mining operations will face the prospect at closure of having to place an engineered cover system over vast areas. Almost all tailings storage facilities (TSFs) and waste rock dumps will require some form of engineered cover, and many of these facilities cover areas of the order of hundreds of hectares. Engineered covers should consist primarily of either selected and stored stockpiled natural soil, or appropriate waste rock (regolith) material that has pedogenic potential. These materials may sometimes include small quantities of rock. This means that required earthwork volumes will typically be of the order of $500,000 \mathrm{~m}^{3}$ or more. To transport, place and spread this volume of material, large earthmoving equipment will invariably be required.

In most soils used for construction of a cover system, it will be impossible to place and spread the soil without causing a relatively high degree of compaction. Even if the soil is placed in relatively thick layers ( $0.5 \mathrm{~m}$ thick), the upper $30 \mathrm{~cm}$ or so will still experience high compaction stresses merely as a consequence of the transferred load of the earthmoving equipment (Scott, 1975).

\section{Compaction due to surface loading}

\subsection{What is compaction?}

In very general terms, compaction is an increase in density of a soil due to the expulsion of air, as a result of the application of energy. Compaction cannot occur in a fully saturated soil, as the water would in this case absorb all the applied energy and no change in volume (increase in density) would occur. Engineered 
compaction is the key to improving the strength and stiffness (resistance to settlement) of many soils used in civil engineering earthworks. The goal of engineered compaction is usually to achieve the maximum possible density for a given input of energy (determined by type of compaction equipment used). A key relationship used in this process is illustrated in Figure 1.

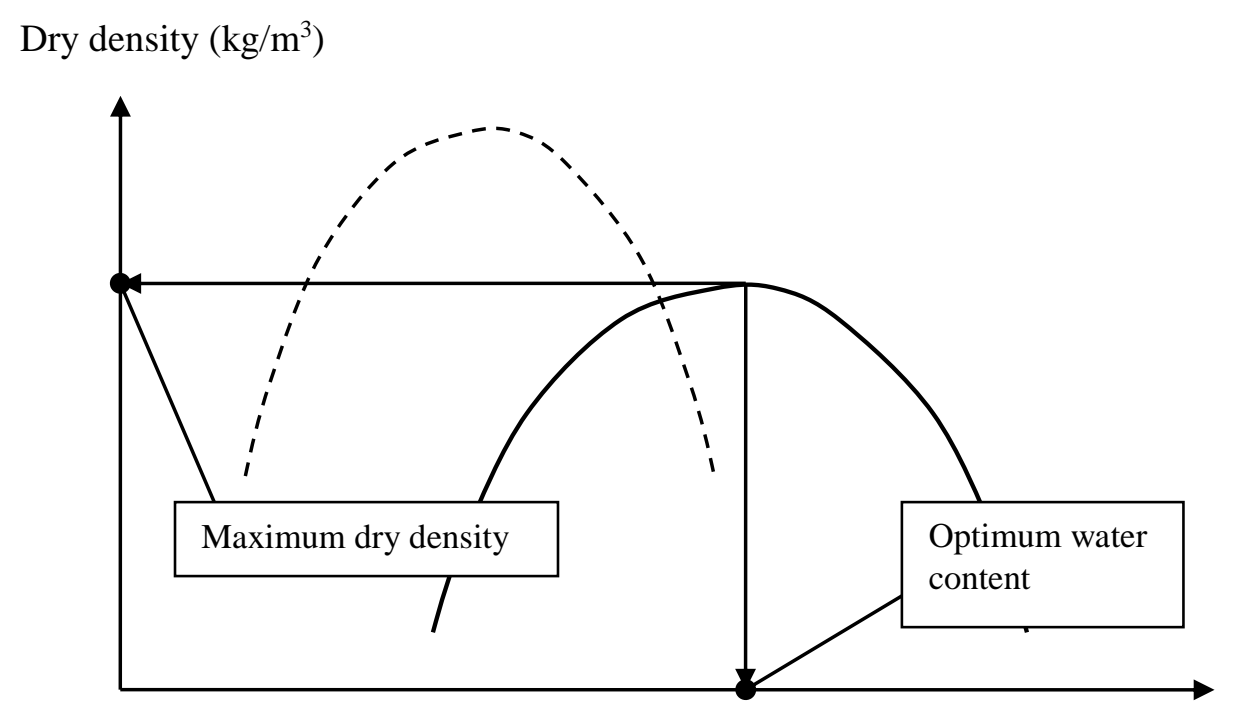

Water content, $\mathrm{w}(\%)$

Figure 1 Illustration of the relationship between water content and dry density for soil subjected to a compaction test

The axes in this figure are the gravimetric water content, $\mathrm{w}\left(M_{w} / M_{s}\right)$, where $\mathrm{M}_{\mathrm{w}}$ is the mass of water and $\mathrm{M}_{\mathrm{s}}$ is the mass of soil, and the dry density, $\rho_{\mathrm{d}}$, not to be confused with the bulk unit weight, $\rho_{\mathrm{b}}$, $\left(\rho_{d}=\rho_{b} /(1+\mathrm{w})\right)$. The dry density is the density of a given volume of soil with all the pore water removed. The solid line parabola on this figure represents a typical relationship between $\mathrm{w}$ and $\rho_{\mathrm{d}}$ for a soil, with this relationship being obtained using a standard testing procedure (e.g. AS1289). As shown, increases in water content result in a greater achievable dry density up to a point, beyond which further increases in water content result in decreasing dry densities. The value of water content at which the maximum dry density is achieved is known as the optimum water content. It is important to realize that (for a given compaction energy), if a soil is compacted at a water content that is either less than or more than the optimum water content (known as the 'dry' side and 'wet' side respectively), it is not possible to achieve the maximum dry density. During engineering compaction projects significant attention is therefore paid to preparing the soil at (or very close to) the optimum water content to ensure the desired densities are achieved.

The dashed parabola in Figure 1 represents a $w-\rho_{d}$ relationship for the same soil as before, but resulting from the application of a higher energy input than before (e.g. by using significantly heavier earthmoving equipment). In this case, a higher maximum dry density is achieved, but at a lower optimum water content. The two curves illustrated in Figure 1 represent target conditions for a particular soil, for two different levels of energy input. In civil engineering parlance these two curves could be thought of as representing the Standard, or Proctor curve (the solid line parabola) and the Modified AASHTO (American Association of State Highway and Transportation Officials) compaction curve (dashed line parabola), where the difference in input energy is of the order of a factor of four. The Standard compaction curve is typically used to provide target values of water content and dry density for applications such as compacted clay liners and covers. The Standard curve was used to provide target densities for the root penetration study reported later in this paper.

There is a great deal of literature dealing with engineered compaction and how best to achieve optimum results for various soil types. In contrast, when considering the construction of an engineered cover system, such as a store-and-release cover, the opposite goal may be paramount, i.e. the prevention of compaction by earthmoving equipment to a density that compromises the cover performance, for example by preventing 
root penetration and thus vegetation establishment. There are some heuristics that indicate that root penetration is not possible in a soil having a bulk density greater than a particular value. However, it is our assertion that guidelines such as these need to be formulated in terms of a more generic framework and we suggest expressing the root penetration resistance density (RPRD), for particular plant growth forms, as a percentage of the Standard compaction maximum dry density. For example, we foresee conclusions such as, 'for soil type $\mathrm{S}$ and plant species $\mathrm{P}$, resistance to root penetration occurs at $93 \%$ of the Standard compaction maximum dry density'. There are clearly too many combinations of soil and plant types that might be used in cover systems to make it feasible to test all the possible combinations, but establishing cutoff values for a few combinations is a necessary first step.

Before describing the experimental programme that considered a particular soil/plant combination, it is necessary to consider why compaction is important, and how surface loads (earthmoving equipment) can be related to changes in density with depth below surface.

\subsection{Why is it important when considering cover systems?}

There is extensive reporting in the literature of compaction (often referred to as densification) having a negative impact on plant development, e.g. Siegel-Issam et al. (2005) describe how compaction caused by intensive forest management practices reduced tree growth rates substantially. The effects of compaction on soil properties can be physical, chemical or biological. The most obvious impact is an increase in soil strength and a consequent reduction in the amount of friable substrate available to plant roots. The increased resistance to root penetration limits root exploration of the regolith, and can significantly alter root architecture (Harrison et al., 1994; Henderson, 1989; Rokich et al., 2001). Reduced root penetration limits the ability of plants to access water stored at depth in the profile, a particularly valuable resource in Western Australian systems, leading to reduced seedling establishment in rehabilitation areas (Rokich et al., 2001). Furthermore, compaction can decrease soil fertility by reducing the store and supply of nutrients and water (Hamza and Anderson, 2005), while reduced oxygen diffusion through the soil profile can result in denitrification (Renault and Stengel, 1994) and decreased micro-organism activity (Hamza and Anderson, 2005).

It has been suggested that the main effect of compaction beyond a critical density is the reduction in large pores (defined as $>30 \mu \mathrm{m}$ ), resulting in decreased values of the soil hydraulic conductivity and surface infiltration rates, reduced soil arthropod and earthworm activity, and altered water retention characteristics (Hakansson et al., 1988). It is also accepted that a soil profile will take a long time to recover naturally from compaction beyond a critical density, if it occurs at all. For example Blake et al. (1976) reported the persistence of increased density in the absence of ploughing. Although it has been suggested (Soane and van Ouwerkerk, 1980) that problems associated with a high degree of compaction are particularly severe where highly mechanized agriculture is practiced in high rainfall areas (presumably because the soil is closer to the optimum water content shown in Figure 1 more often than in dry climates), the problem is one that can be expected to occur in any cover system construction, irrespective of the prevailing climate. This is because the type of equipment likely to be used in construction of these covers will often be significantly heavier than that used in agriculture, from where the vast majority of the literature on over-compaction originates, and thus lower optimum water contents might be relevant.

Although the literature emanating from the agricultural sciences may not always be directly applicable to mining cover systems (as mentioned above), it is a rich source of information and worth considering in some detail.

\subsection{Relationship between equipment type and changes in density below ground surface}

It is clearly an increment in applied stress that will cause a change in volume (densification) of soil below an applied surface load. It is intuitive to expect the magnitude of the stress increase to decrease with depth below the applied surface load, as can be illustrated by reference to an idealized situation indicated in Figure 2 as the solid line. This shows the increment in vertical stress with depth, below the centerline of a circular surface load, expressed as a fraction of the applied surface load. This solution is described in many standard soil mechanic texts (Scott, 1975), and although it represents an idealized condition of a homogeneous, isotropic, elastic medium, it provides a useful point of reference. At the ground surface, directly below the 
applied load, q (in $\mathrm{kPa}$ ), the increase in vertical stress is equal to q. Below the surface it decreases rapidly with depth. For a typical surface contact area of about $900 \mathrm{~cm}^{2}$ (radius of loaded circle equal to $17 \mathrm{~cm}$ ), which is based on a tire width of $30 \mathrm{~cm}$, the increase in vertical stress is $50 \%$ of the applied surface stress at a depth of about $22 \mathrm{~cm}$ below surface, i.e. a relatively thin layer of soil is subject to large stress increases.

However, before going any further, it is important to emphasize the highly idealized condition illustrated in Figure 2. Firstly, it represents conditions below a circular loaded surface area. Truck and other equipment typically have rectangular contact areas. Similar solutions to that illustrated in Figure 2 exist for these conditions, and an example of this is shown for a low pressure dozer track (with dimensions of $3.2 \times 0.57 \mathrm{~m}$ ). It can be seen that the depth at which significant loads are transferred to the soil increases, with the 50\% point now occurring at about $60 \mathrm{~cm}$ depth. In the case of the low pressure dozer considered here, the contact stress was of the order of $82 \mathrm{kPa}$, so a significant increase in stress is transferred through the soil profile, e.g. even at a depth of $1 \mathrm{~m}$, the vertical stress would increase by about $24 \mathrm{kPa}$. Considering the information provided later in this paper (dealing with the effect of load magnitude in addition to ground contact stress), it can be seen that the use of heavy machinery, even when equipped with larger treads, can still be problematical.

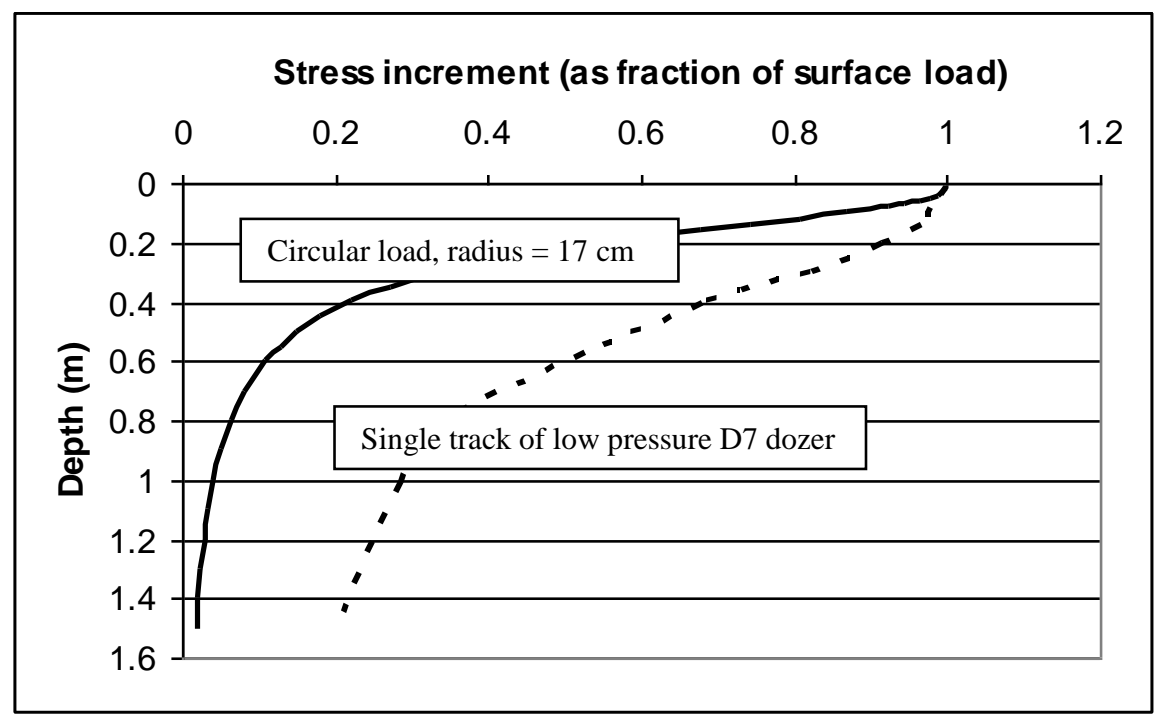
Figure 2 Theoretical increase in vertical stress with depth below centerline of loaded area, expressed
as a fraction of applied surface load

The general conclusions described above are consistent with many field studies reported in the literature. As an example, Dumbeck (1984, as quoted in Hakannson et al., 1988) found increases in density to depth of $1 \mathrm{~m}$ with only $100 \mathrm{kPa}$ ground pressure, but where the total load (in $\mathrm{kN}$ ) was high. Corcoran and Gove (1985) also describe how the effect of tracked vehicles may be worse than thought, probably due to the large surface contact area discussed above, an effect that is exacerbated by uneven pressure distribution between two parallel tracks, the longer duration of loading that results under a tracked load, vibrations that are transmitted through the tracks, as well as the additional loads applied during turning and slewing. The suggested limits that have been set in countries such as Germany $(40 \mathrm{kN})$ and Sweden $(60 \mathrm{kN})$ on individual wheel loads (Hakansson, 1988) to avoid compaction deeper than $40 \mathrm{~cm}$, may be completely irrelevant when conventional mining earthmoving equipment is used during construction of a cover system, even if the equipment is supposedly low contact pressure equipment.

In the current study we have set out to test the relationship between standard engineering compaction curves and the capacity for plants roots to penetrate compacted soil at two contrasting densities on the curve (Figure 1). This was achieved by growing seedlings of a fast growing, short-lived tree species in columns of an appropriate soil material under different levels of compaction and assessing the rooting dynamics under the different treatments. 


\section{Methods and materials}

\subsection{Soil characteristics}

The soil used in this study represented material designated for use as a growing medium on covered tailings storage facilities. It was air-dried for three weeks in a drying room at approximately $35^{\circ} \mathrm{C}$. After drying, it was sieved to less than $6 \mathrm{~mm}$ in size, mainly to remove gravel and wood debris. Index tests on the soil produced the values summarized in Table 1 .

Table 1 Index test properties of soil used in column tests

\begin{tabular}{lc}
\hline Soil Property & Value \\
\hline Specific gravity & 2.61 \\
Liquid limit & $53 \%$ \\
Plasticity index & $27 \%$ \\
Maximum standard dry density & $1840 \mathrm{~kg} / \mathrm{m}^{3}$ \\
Optimum water content & $12.4 \%$ \\
\hline
\end{tabular}

The maximum dry density and corresponding optimum water content were obtained using the test discussed in Section 2.1.

\subsection{Preparation of soil columns}

The soil was packed into $10 \mathrm{~cm}$ diameter, $50 \mathrm{~cm}$ long PVC columns. Packing was achieved using a Standard compaction hammer. The bottom end of each column was sealed with a PVC cap which had been drilled with six holes of $0.5 \mathrm{~cm}$ diameter to provide base drainage. Three different density configurations were used, representing a high degree of compaction $(\mathrm{H})$, low degree of compaction (L) and a composite profile (C). All columns were packed in three layers of equal thickness. Details were:

- H columns were compacted to a uniform dry density of $1650 \mathrm{~kg} / \mathrm{m}^{3}(99 \%$ of standard maximum value), at a water content of $13.9 \%$.

- L columns were compacted to a uniform dry density of $1548 \mathrm{~kg} / \mathrm{m}^{3}$ (93\% of standard maximum value), at a water content of $8.6 \%$.

- $\mathrm{C}$ columns were compacted in three layers, with the upper layer being at the same density and water content as the L columns, as the two lower layers being at the same density and water content as the $\mathrm{H}$ columns. This was done to evaluate the effect of a density change on the development of rooting depth.

Each configuration $(\mathrm{H}, \mathrm{L}$ and $\mathrm{C}$ ) had eight replications, with four of each being dismantled 40 days after first detection of growth, and the other four being dismantled 60 days after detection of first growth.

Acacia celastrifolia, a local native legume, was chosen for use in the study because of its rapid growth habit and large root system. Seeds were sterilized in dilute hypochlorite solution and pre-germinated on $1.5 \%$ water agar plates in a controlled temperature room. An adequate amount $(1.0 \mathrm{~g})$ of a proprietary thrive fertilizer providing a full range of macro and micro nutrients was added to each layer prior to compacting it into the designated column to encourage plant growth. An $8 \mathrm{~cm}$ layer of gravel was placed at the bottom of each column to assist in column drainage. Soil was then compacted into the columns according to one of the three designations listed above. A thin $(3 \mathrm{~cm})$ thick layer of potting mix was spread over the surface of the compacted soil column to provide a bedding layer.

As soon as the seeds had germinated, they were planted into the prepared soil columns by drilling three equi-spaced holes to a depth of $1.5 \mathrm{~cm}$ into the bedding layer. The columns were placed in an air-conditioned glasshouse, with the column arrangement following a completely randomized block design to ensure factors such as sunlight, wind drafts and position had no influence on the test results. 
The start of the growth period was subjectively determined by visual inspection, with day zero being taken as when shoots could be seen to emerge from the growth medium. The columns were watered approximately two to three times per week, depending on the degree of drying that occurred between measurements. The watering schedule for the study was chosen to maintain the columns at $50 \%$ of the total porosity.

\subsection{Harvesting of plants, and measurement of root and shoot responses}

Harvesting occurred at 40 days and 60 days after the first evidence of growth was detected. The abovesurface material (referred to as 'shoots') was cut off and bagged for analysis, the base of the columns cut off and the soil cylinders extruded and bagged. Each of the cylinders was divided into six intervals, with measurements of root depth and length being made from the bottom of the germination layer. The roots were hand-washed until free of all soil and then stored in brown paper bags overnight, before carrying out further measurements.

The roots and shoots were analyzed using a WinRHIZO pattern recognition system, with a resolution of 150 dpi. After scanning, the roots were dried for 24 hours in a $70^{\circ} \mathrm{C}$ oven, to determine their moisture contents. The data are summarized in Table 2, which shows root depth, length and biomass, leaf surface area, shoot biomass, and root-to-shoot ratio. The percentage of the total root mass present in the six depth intervals is shown in Figure 3.

Table 2 Results from harvesting of plants at 40 days and 60 days after detection of first growth. Values are means with standard error in brackets

\begin{tabular}{lcccccc}
\hline & \multicolumn{3}{c}{ Results at 40 days } & \multicolumn{3}{c}{ Results at 60 days } \\
\hline Configuration & H & L & C & H & L & C \\
\hline Average root depth $(\mathrm{cm})$ & $9(4)$ & $178(4)$ & $11(1)$ & $13(0)$ & $39(0)$ & $15(1)$ \\
Total root length $(\mathrm{cm})$ & $283(135)$ & $312(94)$ & $417(79)$ & $1376(48)$ & $2679(263)$ & $2689(233)$ \\
Leaf surface area $\left(\mathrm{cm}^{2}\right)$ & $46(24)$ & $25(6)$ & $34(7)$ & $154(19)$ & $366(24)$ & $341(21)$ \\
Dry root biomass (mg) & $59(15)$ & $67(18)$ & $48(3)$ & $358(91)$ & $753(132)$ & $827(183)$ \\
Shoot biomass (fresh), (mg) & $112(44)$ & $117(33)$ & $121(11)$ & $1190(255)$ & $3174(453)$ & $3518(237)$ \\
Root-to-shoot-ratio & 0.53 & 0.57 & 0.4 & 0.3 & 0.24 & 0.24 \\
\hline
\end{tabular}

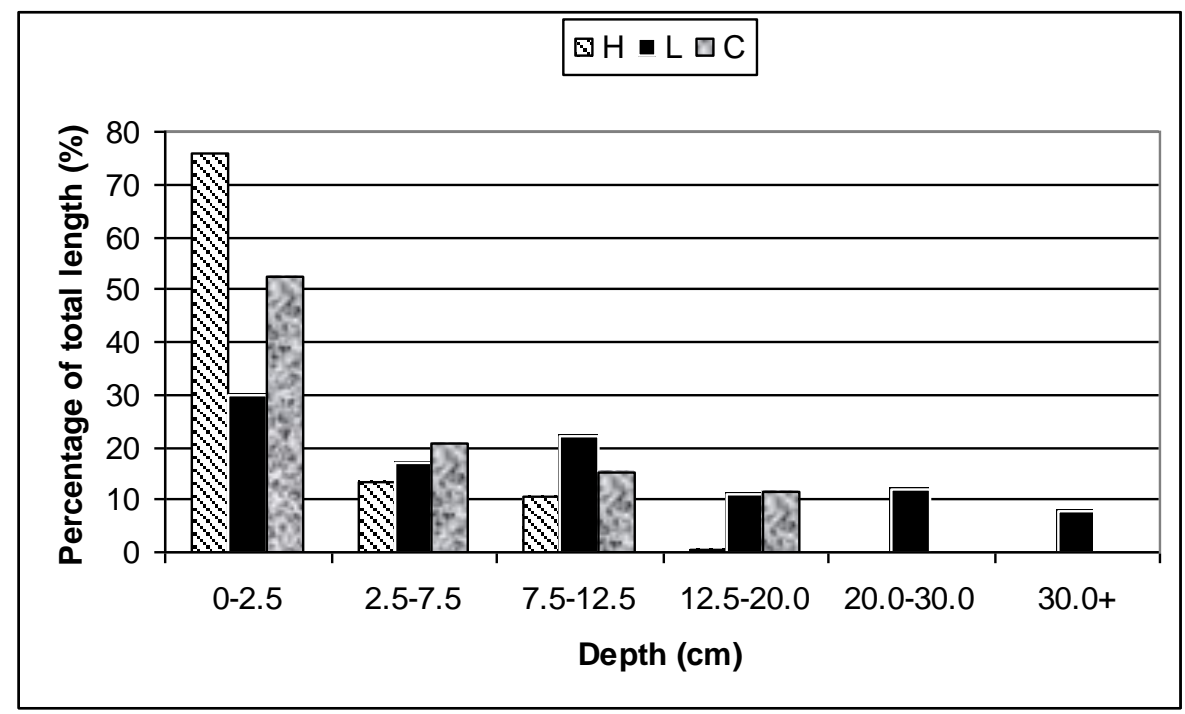

Figure 3 Percentage of total root length occurring within different depth intervals for high density (H), low density (L) and composite (C) compaction profiles. Results were obtained after 60 days of growth 
There are clearly major differences between the three configurations, and it is worth comparing the differences between the high $(\mathrm{H})$ and low $(\mathrm{L})$ compaction density profiles to start with. Considering both Figure 3 and Table 2, it can be seen that the low density profile produces a much greater total root length than the $\mathrm{H}$ profile (about twice as much), with the difference being more pronounced after 60 days than after 40 days of growth. The average root depth is three times greater for the L profile, and as can be seen in Figure 3, the roots proliferated throughout the profile, unlike the $\mathrm{H}$ profile, where they did not penetrate below about $20 \mathrm{~cm}$ depth, and barely reached beyond $12.5 \mathrm{~cm}$. The leaf surface area, root biomass and shoot biomass are also approximately twice as great in the $\mathrm{L}$ profile results at 60 days, with the root-to-shoot ratio accordingly being relatively similar. There is a very high concentration of root development within the upper $2.5 \mathrm{~cm}$ of the $\mathrm{H}$ profile, being almost $80 \%$ of the total root length. Therefore, although the $\mathrm{H}$ profile did sustain vegetation establishment, and the vegetation appeared healthy at the time of harvest, the very shallow rooting system would render any mature vegetation susceptible to drought, uprooting by winds and to exposure by erosion processes in the short term. Access to subsoil water and nutrient stores by shallow rooted vegetation will also be limited.

\section{Results in comparison with previously reported data}

The ability of the vegetation to establish as well as it did in the $\mathrm{H}$ profile is somewhat contradictory to accepted wisdom (generally originating from agricultural sources), where a bulk density of $1700 \mathrm{~kg} / \mathrm{m}^{3}$ is often considered an upper limit for vegetation establishment, whereas the $\mathrm{H}$ profile had a bulk density of $2068 \mathrm{~kg} / \mathrm{m}^{3}$. In fact, even the L profile had a bulk density of $1780 \mathrm{~kg} / \mathrm{m}^{3}$, so it comfortably exceeds the $1700 \mathrm{~kg} / \mathrm{m}^{3}$ limit, but exhibited excellent root development, with relatively uniform spread of roots with depth. The composite (stratified) profile (C), with the low density layer overlying the high density layers produced, as might be expected, a response that is generally somewhere between the other two profiles. There was a greater concentration of roots in the upper $2.5 \mathrm{~cm}$ than in the $\mathrm{L}$ profile, but also a greater distribution with depth than in the H profile (see Figure 3). In some ways, it slightly outperformed the L profile, producing larger root and shoot biomass, although the differences are relatively small. The positive conclusion that might be drawn from this result is that a heavily compacted profile might reasonably be treated by ripping and disturbance to a relatively shallow depth (say $15 \mathrm{~cm}$ ), which is a commonly used technique. Remembering that much of the commonly used earthmoving equipment produces a density profile that decreases rapidly with depth, it might be that the issue is relatively easily addressed. Of course, it remains to conduct experiments with a range of combinations of soil and vegetation type, as the results presented in this paper may represent an isolated case: for example the vegetation chosen may be much more resilient than the norm. Extrapolation to other circumstances is thus inadvisable, but the results are certainly promising in that they indicate current rules of thumb may be unnecessarily restrictive.

\section{Conclusions}

The potential effect of inadvertently compacting soil in a cover system to such a high density that vegetation cannot become established should not be overlooked. The results reviewed in this paper indicate that even earthmoving equipment that is regarded as inducing low ground pressures can actually induce significant increases in density. In addition, when the loaded area is large, such as with dozer tracks, the increase in density can occur to depths of $1 \mathrm{~m}$ or more. However, the commonly accepted limits of allowable bulk density (such as an upper limit of $1700 \mathrm{~kg} / \mathrm{m}^{3}$ bulk density) may be unnecessarily restrictive, and indeed misleading.

In this paper experiments were conducted on columns of a soil compacted to produce different density profiles. A single type of plant, Acacia celastrifolia, was grown in these columns and the root morphology mapped after 40 days and 60 days of growth. The results indicate that growth was achieved in densities in excess of even $2000 \mathrm{~kg} / \mathrm{m}^{3}$ bulk density, although better results were achieved at a lower density.

An important factor discussed in the paper is the use of a standard method of comparing density profiles. The procedure adopted in this paper was the use of the standard compaction curve as the reference framework, which is a logical approach as all earthmoving quality control uses this same procedure in controlling earthworks placement and compaction. It is suggested that this framework be adopted for future work to provide a rational means of comparing data from different studies. 


\section{References}

Australian Standard AS1289. Methods of Testing Soil for Engineering Purposes.

Blake, G.R., Nelson, W.W. and Allmaras, R.R. (1976) Persistence of subsoil compaction in a mollisol. Soil Sci. Soc. Am. J., 40: pp. 943-947.

Corcoran, P.T. and Gove, D.S. (1985) Understanding the mechanics of track traction. Proc. Int. Conf. on Soil Dynamics, 17-19 June, Auburn University, Auburn, Alabama, U.S.A., pp. 664-678.

Dumbeck, G. (1984) Influence of extremely high vehicular loads on soil structure and root development. Mitt. Dtsch. Bodenkundl. Ges., 40, pp. 61-62 (in German).

Hakansson, I., Voorhees, W.B. and Riley, H. (1988) Vehicle and wheel factors influencing soil compaction and crop response in different traffic regimes. Soil and Tillage Research, 11: pp. 239-282.

Hamza, M.A. and Anderson, W.K. (2005) Soil compaction in cropping systems - a review of the nature, causes and possible solutions, Soil and tillage research, 82, pp. 121-145.

Harrison, D.F., Cameron, K.C. and McLaren, R.G. (1994) Effects of subsoil loosening on soil physical-properties, plant-root growth, and pasture yield, New Zealand Journal of Agricultural Research, 37, pp. 559-567.

Henderson, C.W.L. (1989) Using a penetrometer to predict the effects of soil compaction on the growth and yield of wheat on uniform, sandy soils, Australian Journal of Agricultural Research, 40, pp. 497-508.

Renault, P. and Stengel, P. (1994) Modeling oxygen diffusion in aggregated soils, 1. Anaerobiosis inside the aggregates, Soil Science Society of America Journal, 58, pp. 1017-1023.

Rokich, D.P., Meney, K.A., Dixon, K.W. and Sivasithamparam, K. (2001) The impact of soil disturbance on root development in woodland communities in Western Australia, Australian Journal Of Botany, 49, pp. 169-183.

Scott, C.R. (1975) An introduction to soil mechanics and foundations. Applied Science Publishers.

Siegel-Issam, C.M., Burger, J.A., Powers, R.F., Ponder, F. and Patterson, S.C. (2005) Seedling Root Growth as a Function of Soil Density and Water Content. Soil Science Society of America Journal; Jan/Feb 2005, 69, 1, pp. 215-226.

Soane, B.D. and Van Ouwerkerk C. (1980) The role of field traffic studies in soil management research, Soil and Tillage Research, 1, pp. 205-206. 